\title{
On a dead female hookworm, probably Ancylostoma tubaeforme, from the vitreous of a patient in Sri Lanka
}

A. S. Dissanaike', R. L. Thalamulla ${ }^{2}$, Daya de Silva ${ }^{3}$, Saliya Pathirana ${ }^{4}$, Udaya Weerakoon ${ }^{3}$,

M. S. Amaratunga ${ }^{3}$ and T. de S. Naotunna ${ }^{2 *}$.

The Ceylon Journal of Medical Science 2000; 43: 25-30

\section{Summary}

A portion of a female hookworm identified as a species of Ancylostoma was recovered from the vitreous of a patient in Sri Lanka. The worm was dead with a cellular tissue reaction around the anterior part of the body. The internal structure of the worm, which was severed at the anterior end, was not clearly discernible but the characteristic mucron at the tip of the tail confirmed that it is an Ancylostoma sp. In the absence of the mouth end it is not possible to clearly identify it to the species, but it is believed to be $A$. tubaeforme based on its width, short tail, thicker cuticle, and the space between transverse striations.

The probable mode of infection of the patient is discussed.

The patient had scanty hookworm ova in his stools but only a single female Necator americanus was recovered after treatment with anthelmintics.

An incidental observation of some significance was the finding of zoonotic Ancylostoma ova in the patient's son's stools, which needs further investigation.

\section{Introduction}

A few larval nematodes such as Toxocara spp. and larval hookworms have been reported from the human eye (1). More numerous are records of adult filarial worms and of larval and juvenile rodent lungworms from the eye $(2,3,4,5,6)$. However, this is the first time that an adult hookworm of animals, albeit dead, has been seen in the human eye.

\section{Case Report}

In January 2000, a 46 year old man from Panadura, in the Western Province of Sri Lanka, presented to the Eye Hospital, Colombo with a history of floaters of two months duration and gradually deteriorating vision for one month. His right eye vision was positive only for perception of light, and his left eye vision was $6 / 6$.

On examination the right eye showed signs of panuveitis with redness and ciliary injection with keratic precipitates, inflammatory cells in the anterior chamber, posterior synechiae with a small pupil and many inflammatory cells and opacities in the vitreous. He was immediately admitted, started on topical steroids and mydriatics and investigated for panuveitis the common causes for which were negative.

A few days following steroid treatment the anterior chamber and the vitreous became clearer as the inflammatory cells and opacities became less. Following breaking up of posterior synechiae, full dilatation of the pupil was possible with mydriatics showing few pigment deposits on the anterior surface of the lens (Fig 1). The fundus view also became clearer with less inflammation and full dilatation of the pupil. On examination the inferior fundus revealed a large tear and a detachment of the surrounding retina. To a strand of the detached retina was attached a dead worm projecting into the vitreous from the site of the tear with part of the body entrapped in "fibrous" tissue (Fig. 2).

1. 28, Welikadewatte, Nawala Road, Rajagiriya, Sri Lanka.

2. Department of Parasitology, Faculty of Medicine, University of Colombo, Colombo 8, Sri Lanka.

$2^{*}$ (Presently at 3/31, Hanover Street, Wades Town, Wellington, New Zealand).

3. Ward 15, Eye Hospital, Colombo, Sri Lanka.

4. Vitreoretinal Unit, Eye Hospital, Colombo, Sri Lanka. 


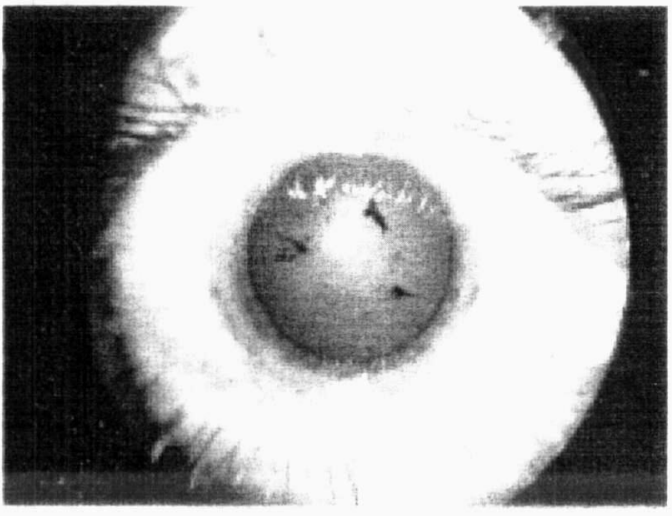

Fig 1: Right eye of the patient with dilated pupil showing pigment on anterior surface of the lens

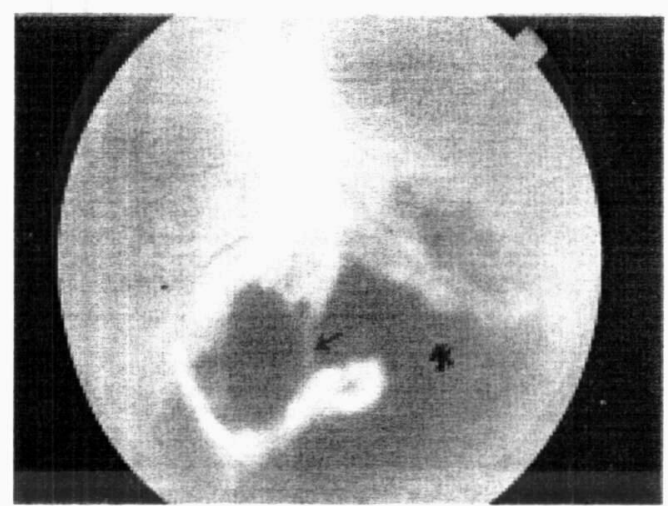

Fig 2: Fundus showing the curved anterior end of the worm attached to a strand of the detached retina (arrow); The exposed choroid is also seen $\left({ }^{*}\right)$

The patient was referred to one of us (SP), who performed trans pars plana vitrectomy under general anaesthesia with the removal of part of the worm that was projecting into the vitreous. The sub retinal fluid under the detachment was drained. The detached retina was flattened with an encircling band applied around the globe and the vitreous cavity filled with silicone oil. Two days later laser burns were applied around the retinal tear to prevent another detachment. There was no subjective improvement of vision following surgery probably due to the presence of the detached macula and large area of atrophic retina.
The part of the worm that was removed was sent for identification to the Department of Parasitology, University of Colombo.

The patient who is an alcoholic did not complain of any significant illness in the past. He is married and has four children. He works as a labourer and his main income is from plucking coconuts and cleaning of septic tanks and soakage pits.

Stool examination of the patient showed Trichuris trichiura ova and scanty hookworm ova only on salt floatation but the stool culture was negative. Stools of the household cat and a neighbour's dog, which frequently visits the patient's home, were positive for hookworm ova and cultures showed Ancylostoma larvae. The 18 year old son's stool was also positive for hookworm ova and culture revealed Ancylostoma larvae, but he refused anthelmintic treatment.

The patient was treated with two courses of anthelmintics, first albendazole $600 \mathrm{mg}$ in a single dose and subsequently, a week later, single dose of $700 \mathrm{mg}$ mebendazole followed by a magnesium sulphate purgative. Apart from several Enterobius vermicularis adult worms only a single female Necator americanus was recovered from the patient's stool after treatment.

Other laboratory investigations:

WBC/DC $8,400 / \mathrm{mm}^{3}$ - neutrophils $70 \%$, lymphocytes $27 \%$, eosinophils $2 \%$, monocytes $1 \%$,

HB $-14.9 \mathrm{~g} / \mathrm{dL}$

E.S.R. $10 \mathrm{mn} 1^{\text {st }} \mathrm{hr}$.

\section{Description of the worm}

An outline drawing (Fig. 3) shows the cellular tissue reaction surrounding the worm to occupy a length of $4.5 \mathrm{~mm}$. As the anterior portion of the worm is missing the remaining part is only 7.3 $\mathrm{mm}$ with an average width of $390 \mu \mathrm{m}$. The fundus photograph shows the worm to have an apparent length of only $6 \mathrm{~mm}$ including the curved anterior end. However this measurement is based 
on the assumption that the disc is $1.5 \mathrm{~mm}$ and it also appears much smaller since a portion of the worm is seen end on projecting forward towards the vitreous. The curved anterior portion of the worm seems to be about $20 \%$ of the entire length measured by this method suggesting that the actual length must have been around $9.1 \mathrm{~mm}$.

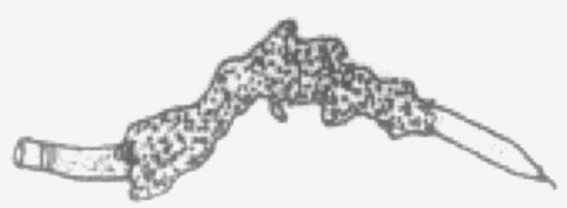

1

Fig 3: Line drawing of the part of the worm that was recovered at operation showing the tissue reaction (scale bar $=5 \mathrm{~mm}$ )

The tail end of the worm, which is clearly a female, shows the mucron $20 \mu \mathrm{m}$ in length. The characteristic transverse cuticular striations are $6.4-6.9 \mu \mathrm{m}$ in width and the cuticle thickness is $9.3-14.5 \mu \mathrm{m}$ posterior to the anus (Fig. 4). No details of the internal structure of the worm are discernible and the position of the vulva cannot be identified clearly. The tail is $156 \mu \mathrm{m}$ and is curved ventrad (Fig. 5).

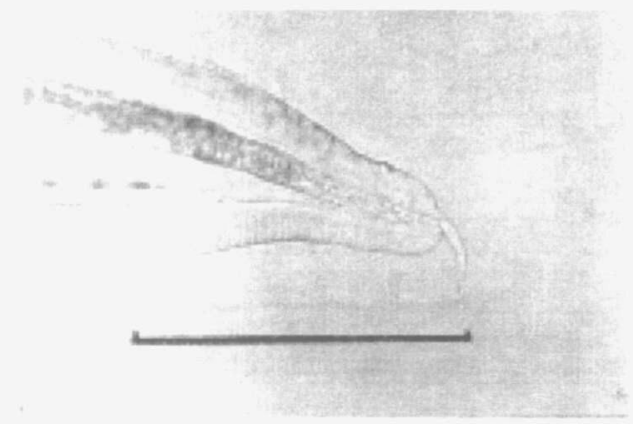

Fig 4: Photomicrograph of the tail end showing the characteristic mucron, transverse striations and thickness of the cuticle (Scale bar $=100 \mu \mathrm{m}$ )

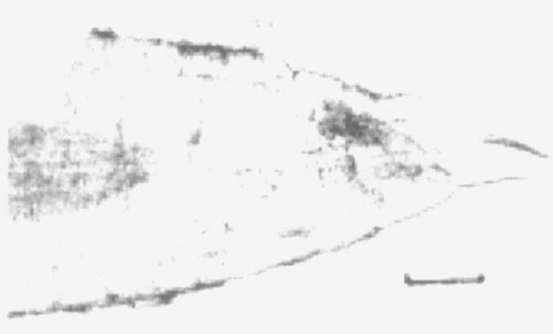

Fig 5: Tail end of the worm showing ventrad curvature (Scale bar $=100 \mu \mathrm{m}$ )

\section{Discussion}

The larval nematodes that have been reported from the human eye include Toxocara spp. of dogs and cats (1) and larvae tentatively identified as hookworm larvae (7). Many juvenile and adult nematodes have been recorded on numerous occasions and have been reviewed and discussed. They include the filarial worms Wuchereria bancrofti (8) from the anterior chamber and in recent years from the conjunctiva (9); Dirofilaria (Dirofilaria) immitis (3) from the vitreous; Dirofilaria (Nochtiella) repens $(10,4)$ from the conjunctiva and the rodent lungworms Parastrongylus (=Angiostrongylus) spp. $(5,6,11)$ from the vitreous and anterior chamber.

However, this is the first record of an adult hookworm from the human eye. The tail end of the specimen, which was free of cellular debris, showed transverse cuticular striations characteristic of the genus Ancylostoma. It cannot be $A$. duodenale since that parasite is not found in humans in Sri Lanka and is also a much stouter worm. It could therefore be one of the zoonotic species of cats or dogs which usually cause infection by the oral route. The common species reported from Sri Lanka are: $A$ caninum, $A$. braziliense and $A$. ceylanicum from dogs $(12,13,14)$; and $A$. braziliense and $A$. caninum (now regarded as $A$. tubaeforme, $(15,16)\}$ from cats $(12)$.

In the absence of the mouth end with the teeth it is not possible to identify the present worm clearly 
to the species. The smaller width, shorter tail and wider spaces between the cuticular striations would suggest that it is not $A$. caninum $(15,16,17$, 18). Various available measurements of the specimen seem to overlap with those of $A$. braziliense, $A$. ceylanicum (19) and $A$. tubaeforme $(15,16)$. The thicker cuticle, the abruptly narrowing tail posterior to the anus and its ventrad curvature suggest that it is $A$. tubaeforme $(15,16,17)$. These findings are confirmed by electron microscopy studies (18, 19). But as pointed out by Yoshida (20) "Size and proportions of the body are not distinctive" and hence it is not possible to definitely decide between these species.

We, can only speculate as to how an adult Ancylostoma came to lie in the vitreous. Two possibilities were discussed to explain the presence of $D$. immitis in the vitreous (3): (a) the worm came through the blood stream to the choroid and then to the vitreous in the macular region: or (b) from the anterior chamber to the posterior chamber through the ciliary body into the vitreous.

Canine Ancylostoma spp. have been shown experimentally in rodents to move to extra intestinal sites after oral or cutaneous infection but they have always been encysted either in muscle or more rarely in other sites like the brain, but as third stage larvae $(21,22)$. It is unlikely that a third stage larva reached the retina through the blood stream and then developed to the adult stage. This is all the more so as the patient did not appear to have an Ancylostoma sp. in his intestine, only a single female $N$. americanus being recovered from the stools after anthelmintic treatment. On the other hand his occupation and relatively unhygienic habits and his close association with the pet cat and the neighbour's dog suggest the possibility of an adult Ancylostoma from one of these animals being deposited and contaminating his skin especially in the region of the eyes and boring its way into the anterior chamber. Against this is the fact that the comea is too thick for penetration unless a weak spot is available in the limbus area from which the worm found its way. The most likely route of entry is for an adult worm to have entered directly into the eye possibly through the bulbar conjunctiva (with or without previous in- jury) and sclera either via Schelmn's canal or the aqueous veins into the suprachoroidal space. On entering the vitreous through the retina with the aid of its buccal teeth, its subsequent attachment to the retina would have been the cause of the retinal detachment.

The patient may have had some trauma to the cornea which he was not aware of, although he denied having experienced any discomfort, irritation or redness of the eyes prior to losing his vision.

Although the patient did not show Ancylostoma larvae on stool culture, his 18 year old son's stools were positive for hookworm ova and on culture showed Ancylostoma larvae. Unfortunately he refused anthelmintic treatment. In any event the fact that he has Ancylostoma infection shows that zoonotic Ancylostoma infection is probably not uncommon in Sri Lanka and it is important that this be followed up. It is not likely that he has $A$. caninum in his intestines as this worm is generally immature in humans (23). Nor could his infection be with $A$. braziliense as this parasite has not hitherto been seen in the adult stage in humans (20). The human pathogenecity of $A$. tubaeforme is also unknown nor is its infectivity to humans (23). Hence his infection is most probably with $A$. ceylanicum. Thus it is possible that $A$. ceylanicum in particular is present in humans in Sri Lanka just as it is in India, West New Guinea, Taiwan and the Philippines as discussed recently by Choo et al (2000) (24). In fact $A$. ceylanicum, (Loos 1911) first described from a civet cat in Sri Lanka was shown by Kannangara and Karunaratne (14) to have a high prevalence of $92 \%$ in dogs and occurred in greater numbers than other species of Ancylostoma.

This case is unique in that it is the first time that an adult Ancylostoma has been seen in the human eye. It is unfortunate that the mouth end with the teeth was not available to make a definite identification of the parasite to the species. Recently diffuse unilateral subacute neuroretinitis has also been attributed to Ancylostoma spp. (25). However, the measurements of the worms pictured in their publication clearly suggest that there are larval stages. 


\section{Acknowledgements}

We wish to thank Mr. R. A. A. N Ranawaka, Photography Unit, Department of Pharmacology, Faculty of Medicine and the following from the Department of Parasitology, Faculty of Medicine:

Mrs. H. K. Wickramathanthri for help in the preparation of the manuscript, Miss H. B. R. Sajeewani for technical support and Mr. P. A. G. Anura for his assistance.

\section{References}

1. Beaver, PC. Parasitological Reviews. Larva migrans. Experimental Parasitology 1956; 5: 587-621.

2. Dissanaike AS, Quah Cheng Hock, Then Suke Min. Mature female filaria, probably Brugia sp., from the conjunctiva of man in West Malaysia. American Journal of Tropical Medicine and Hygiene 1974; 23: 1023-26.

3. Dissanaike AS, Ramaligam $S$, Fong A, Pathmayokan S, Thomas V, Kan SP. Filaria in the vitreous of the eye of man in Peninsular Malaysia. American Journal of Tropical Medicine and Hygiene 1977; 26: 1143-47.

4. Dissanaike AS, Abeyewickreme W, Wijesundera M DeS, Weerasooriya M, Ismail MM. Human dirofilariasis caused by Dirofilaria (Nochtiella) repens in Sri Lanka. Parassitologia 1997; 39: 375-82.

5. Durette-Desset MC, Chabaud AG, Cassim MHS, Ismail MM, Premaratne UN, Abeyewickreme W, Dissanaike AS. On an infection of the human eye with Parastrongylus (=Angiostrongylus) sp. in Sri Lanka. Journal of Helminthology 1993; 67: 67-72.

6. Wariyapola $D$, Goonesinghe $\mathrm{N}$, Hsu Priyanna TH. Fonseka C, Ismail MM, Abeyewickrema W, Dissanaike AS. Second case of ocular parastrongyliasis from Sri Lanka. Transactions of the Royal Society of Tropical Medicine and Hygiene 1998; 92: 64-65.
7. Wilder HC. Nematode endophthalmitis. Transactions of the American Academy of Ophthalmology and Otolaryngology 1950; 55: 99-109.

8. Fernando SE. Ocular filariasis: (adult Wuchereria bancrofti in the anterior chamber of human eye). Journal of Tropical Medicine and Hygiene 1935; 38: 17-18.

9. Gautret P, Bain O, Gicquel JJ, Hue B, Kauffmann-Lacroix C, Rodier MH, Sinnaeve $O$, Jacquemin JL. Localisation sous-conjonctivale d'une femelle adulte de Wuchereria bancrofti. Bulletin de la Societe de Pathologie Exotique et de ses filiales 1999; 92: 104-6.

10. Pampiglione S, Canestri Trotti G, Rivasi F. Human dirofilariasis due to Dirofilaria (Notchiella) repens: a review of world literature. Parassitologia 1995; 37: 149-193.

11. Dissanaike AS, Ihalmulla RL, Naotunne T De $S$, Senarathna T, Witharana DSR. Third report of ocular parastrongyliasis (angiostrongyliasis) from Sri Lanka. Parassitologia. (2000 in Press)

12. Seneviratne P. A checklist of helminths in the Department of Veterinary Pathology, University of Ceylon Peradeniya. Ceylon Veterinary Journal 1955; 3: 32-37.

13. Dissanaike AS. On some helminths of dogs in Colombo and their bearing on human infections, with a description of a new trematode Heterophyopsis yehi sp. nov. (Heterophyidae). Ceylon Journal of Medical Science 1961; 10: 1-12.

14. Kannangara DWW, Karunaratne GMS. A note on intestinal helminths of dogs in Colombo. Ceylon Veterinary Journal 1970; 18: 47-49.

15. Biocca E. Ridescrizione di Ancylostoma tubaeforme (Zeder, 1800) Parassita del Gatto, Considerato erroneamente Sinonimo di Ancylostoma caninum (Ercolani, 1859), Parassita del cane. Rivista di Parassitologia 1954; 15: 267-278. 
16. Burrows RB. Comparative morphology of Ancylostoma tubaeforme (Zeder, 1800) and Ancylostoma canninum (Ercolani, 1859). Journal of Parasitology 1962; 48: 715-718.

17. Lane C. The genus Ancylostom in India and Ceylon. Indian Journal of Medical Research 1916; 4: 74-92.

18. Innocenti L, Macchioni G. Osservaziono su Ancylostoma canninum ed $A$. tubaeforme al microscopio ottico ed al microscopio elettronico a scansione. Annali della Facolta' di medicina veternaria. Universita' di Pisa 1990; 43: 127-135.

19. Yoshida Y, Comparative studies on Ancylostoma braziliense and Ancylostoma ceylanicum 1. The adult stage. Journal of Parasitology 1971; 57: 983-989.

20. Yoshida Y. Ancylostoma kusimaense from a dog in Japan and comparative morphology of related ancylostomes. Journal of Parasitology $1965 ; 61: 631-635$.
21. Norris $\mathrm{DE}$. The migratory behaviours of the infective larvae of Ancylostoma braziliense and Ancylostoma tubaeforme in rodent paratenic hosts. Journal of Parasitology 1971; 57: 9981009.

22. Yoshida $Y$, Kondo K, Kurimoto H, Fukutome $\mathrm{S}$, Shirasaka S. Comparative studies on Ancylostoma braziliense and A. ceylanicum III. Life history in the definitive host. Journal of Parasitology 1974; 60: 636-641.

23. Prociv $P$, Croese J. Human enteric infection with Ancylostoma caninum: hookworms reappraised in the light of a "new" zoonosis. Acta Tropica 1996; 62: 23-44.

24. Choo J, Pang E, Prociv P. Hookworms in dogs of Kuching, Sarawak (North Borneo). Transactions of the Royal Society of Tropical Medicine and Hygiene 2000; 94: 21-22.

25. Cunha De Souza E, Lustosa Da Cunha S, Donald J. Gass M. Diffuse unilateral subacute neuroretinitis in South America. Archieves of Ophthalmology 1992; 110: 1261-1263. 